\title{
Analyzing Costs of Space Debris Removal in Basis of Three Kinds of Methods
}

\author{
Jialin Wen ${ }^{1}$ \\ ${ }^{1}$ Electrical and Information College, Jinan University, China \\ Correspondence: Jialin Wen, Electrical and Information College, Jinan University, Qianshan Road 206, Zhuhai, \\ China. Tel: 96-158-2057-8305. E-mail: 1094886064@qq.com
}

Received: May 24, 2017

Accepted: June 12, 2017

Online Published: July 20, 2017

doi:10.5539/ijef.v9n8p151

URL: https://doi.org/10.5539/ijef.v9n8p151

\begin{abstract}
The number of different space debris on the orbits around the earth has gotten more and more attentions. These debris pieces put spacecraft in a dangerous place. We built a model based on the problems of the space debris' removal, discussing the cost of the three common methods (ground-based laser removing, water jet cutting and net-capture) for removing the debris. First we used the Monte Carlo to simulate the space debris' number and speed. Then we built models for the three common methods respectively, explored the removing efficiency of different size of debris and their cost for removing a unit volume of the debris. Finally we find that The laser removing method is suiTable for medium-sized debris with the diameter less than $10 \mathrm{~cm}$, the water jet method is suiTable for large-sized debris with the diameter within $10 \mathrm{~cm}$ and $1400 \mathrm{~cm}$, the net-capture method is suiTable for super-large-sized debris with diameter bigger than $1400 \mathrm{~cm}$. Also, we analyzed the advantages and shortcoming of each method.
\end{abstract}

Keywords: space debris, ground-based laser removing, water jet cutting, net-capture, cost

\section{Introduction}

Space Junk The amount of small debris in orbit around earth has been a growing concern. It is estimated that more than 500,000 pieces of space debris, also called orbital debris, are currently being tracked as potential hazards to space craft. The issue itself became more widely discussed in the news media when the Russian satellite Kosmos-2251 and the USA satellite Iridium-33 collided on 10 February, 2009. A number of methods to remove the debris have been proposed. These methods include small, space-based water jets and high energy lasers used to target specific pieces of debris and large satellites designed to sweep up the debris, among others. The debris ranges in size and mass from paint flakes to abandoned satellites. The debris' high velocity orbits make capture difficult. So we built a model discussing the cost of the three common methods (ground-based laser removing, water jet cutting and net-capture).

At present, Tong Guoliang have analysed the Present situation of space debris and future. Li Chunlai and other scholars have searched for the space environment. Wang Ruihe and other scholars have studied on The rock breaking mechanism and further studied on high pressure water jet. Zhou Wenhui has created a numerical simulation of internal and external flow field of high pressure water jet nozzle. Scholar Tao Bin has researched on the theory and technology of high pressure water jet processing. Zhu Yilin have Modeled and analysed the space debris environment in low earth orbit. Chang Hao and other scholars have analysed the debris process in the ground laser cleaning process. Li Gensheng and other scholars have discussed rock damage and fracture mechanism under the action of super high pressure water jet. Ni Hongiian and other scholars have considered space net expansion of projectile dynamics.

Since we don't have the data of the space debris, what we need to do first must be the simulation of different debris' quantity increment and speed. We could get the annual increment in quantity of different sized debris. There is a variety of the space debris, in order to simplify the calculation, we assume the space debris as aluminum plates with $1 \mathrm{~cm}$ of thickness.

Since the removing methods aren't clear, we then build models for the three methods exploring their efficiency in capturing different sized debris and the cost in removing every unit volume of the debris. Then analyze the pros and cons of each method clearly. 


\section{Model Preparation}

\subsection{Theory of Space Debris Removal Using Ground-Based Lasers}

Under the single pulse laser's ablation effect, the speed increment of the debris could be seen as instant and the debris' orbit change during the pulse affecting time could be ignored. However, the orbit change during the pulse interval must not be ignored.

The debris travels during the laser pulse intervals and changes its orbit instantly when it gets the laser pulse irradiation and then it travels through the connection between the earth's core and the laser. the process above is called one pass. If the debris' orbit's lowest height reaches the critical altitude of the dense atmosphere burnout when the height reaches $100 \mathrm{~km}$. before the next one pass, the process is called one pass, in de-obit. Generally, after every one pass, if the debris' orbit's lowest height hasn't reached the critical height. Thus, multiple passes are required until debris burnt.

\subsection{Theory of Space Debris Removal Using Space-Based Water Jets}

The high-pressured water jet cutting is a special way of removal. The water jet could reach the pressure up from $10 \mathrm{MPa}$ to $400 \mathrm{Mpa}$ or even higher with the help of superchargers. The water gains pressure energy and squirts out of tiny injectors, then the pressure energy transforms into kinetic energy therefore forms a high speed jet-flow. The water jet removal reaches its goal of removing the debris by using this kinetic energy of the high speed jet-flow to shock and do damage to the workpieces.

\subsection{Theory of Space Debris Removal Using the Space Net-Capturing Large Satellites}

\subsubsection{General Description of the Theory}

There are multiple ways of removing using large satellites, this article would take the net-capture as an example to roughly introduce the method of large satellite removing. In order to make the removing more convenient, we think that the satellite should be capable of changing velocity and the maximum velocity must be faster than the space debris' maximum velocity.

Generally the net-capture system is formed of carrier spacecraft, net casting system, net and the four towing pieces in its four corners. In the parameter range we set, changing the casting parameter, which are the towing piece's mass, the casting angle, the casting speed, we could discover the optimal casting parameter. In the condition of gathering the same number of debris pieces, only when the casting system uses the optimal parameters would the cost be the lowest.

\subsubsection{Performance Parameters of the Net}

The net system pops out and spreads open through the towing of the towing pieces, then operates capturing and brings the object back or away from its orbit compulsively. In order to measure its performance in spreading, capturing and the distance, define the net-capture performance parameters as:

The maximum spreading area $A_{\max }$ : The maximum spreading area of the net.

Invalid capture distance $d_{l}$ : The distance the carrier spacecraft travel where the net spread from $0 \%$ to $80 \%$ of its maximum area (the distance from the towing pieces' mass center and the net's center point to the coordinate system's origin).

The area at the predicted position $A_{d}$ : The area of the net at the predicted capture distance.

The time at the predicted position $t_{d}$ : Time need at reaching the predicted capture distance.

The distance at the maximum area $d_{A \max }$ : The distance traveled by the spacecraft where the net spreads to its maximum area.

\subsection{Parameters of Debris(Al)}

According to the reference, aluminum is a typical of space debris material, by referring to the introduced mass model and the relating parameters, it could be presented as the Table 1:

Table 1. Parameters of aluminum space debris

\begin{tabular}{lcccc}
\hline Description & Mass/g & Diameter $/ \mathrm{cm}$ & Ablation rate $/\left(\mu g \cdot J^{-1}\right)$ & Optimum impact coupling coefficient $\left(d y n \cdot s \cdot J^{-1}\right)$ \\
\hline Al sphere & 78.5 & 10 & 80 & 2 \\
\hline Note. dyn $=10^{-5} \mathrm{~N}$ & & &
\end{tabular}




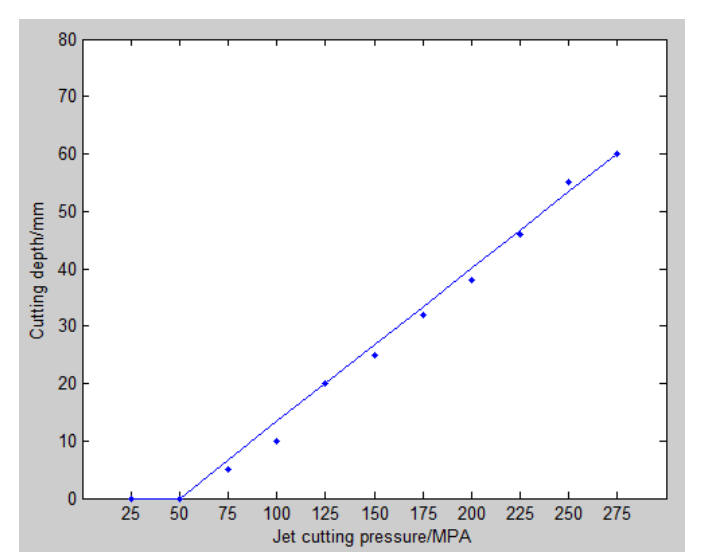

Figure 1. Jet cutting pressure and cutting depth (Al)

\section{Method}

\subsection{Simulation of the Space Debris}

NASA estimates that there are about 500,000 pieces of the debris around the earth's surface with the diameter fixed between 1 centimeter and 10 centimeters, and even more than 100 million pieces of granular debris with diameters less than 1 centimeter. Pieces of larger-sized debris increase around 225 per year, and the medium-sized debris increase around 3375 per year.

Since the space devices debris shield could only protect the workpiece from the space debris with diameters less than 1 centimeter, we only take large-sized and medium-sized debris' removal into concern.

Using the simulation based on Monte Carlo algorithm, with the low orbit height of 100 to $1500 \mathrm{~km}$, the simulation period is set in 1950 to 2008 . And the result of the space debris increment is as followed:

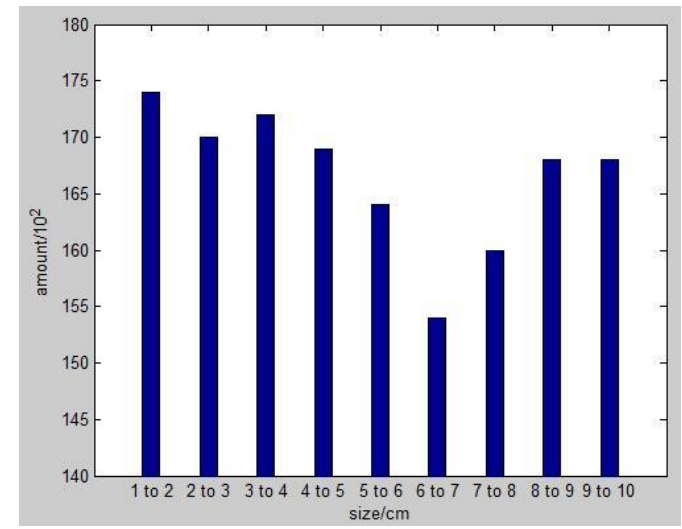

Figure 2. The number of space debris

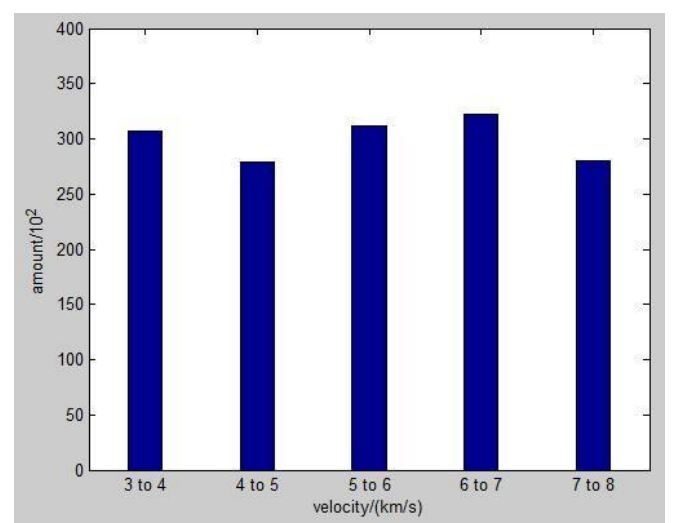

Figure 3. The speed of space debris

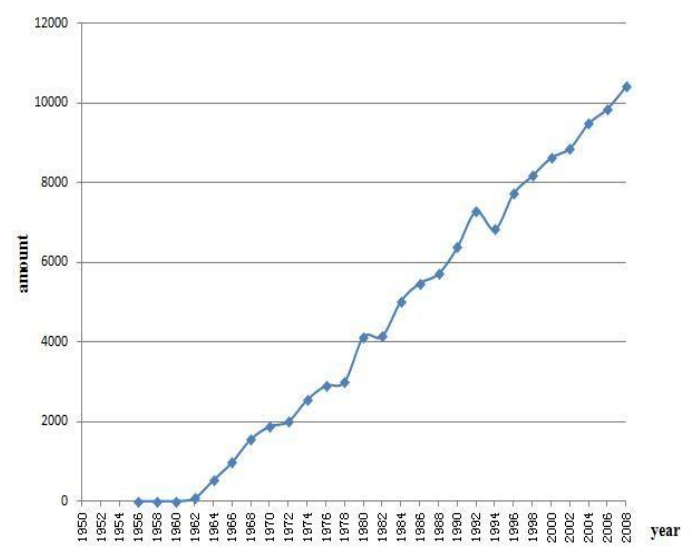

Figure 4. The increase of space debris 


\subsection{Cost Estimation of the Ground-Based Lasers}

We analyzed the principle of the ground laser cleaning space debris, and established a dynamic model of debris removal process. In this paper, a typical laser is selected to simulate the removal effect of space debris.

\subsubsection{Removal Performance Using the Ground Based Laser}

The ground based laser's parameters before the orbit change are set before the $m$-th laser pulse activates, the debris travels on the elliptical orbit $S$, the eccentricity is $e_{s}$, the perigee radius is $r_{p s}$, then the elliptical orbit's semi-major axes is:

$$
a_{s}=\frac{r_{p s}}{1-e_{s}}
$$

- When the debris travels to point $\alpha$, the orbit height is $H_{\alpha}$, then in the point $\alpha$ the orbit radius is $r_{\alpha}$, the speed is $v_{\alpha}$, the local velocity inclination angle is $\beta_{\alpha}$, equation is:

$$
\begin{gathered}
r_{a}=R+H_{a} \\
v_{a}=\sqrt{\frac{2 \mu}{r_{a}}-\frac{\mu}{a_{s}}} \\
\beta_{a}=\arccos \sqrt{\frac{e_{s}^{2}-1}{\frac{v_{0}}{\mu / r_{a}}\left(\frac{v_{a}}{\mu / r_{a}}-2\right)}}
\end{gathered}
$$

In the equation: $R$ and $\mu$ represents the earth's radius and gravitational constant.

- Calculation of speed change caused by the laser pulse irradiation

At point $\alpha$, the space debris gets the laser pulse irradiation, gains the speed increment.

In the Figure: $\xi_{a}$ represents the zenith angle, which is the irradiation angle. According to the geometrical relation shown in the Figure, we could get:

$$
\xi_{a}{ }^{\prime}=\xi_{a}-\arcsin \left(\frac{R}{r} \sin \xi_{a}\right)
$$

In the equation: $\xi_{a}$ and $\xi_{a}^{\prime}$ are of the same sign and $\xi_{a}>\xi_{a}^{\prime}$. The radial and axial speed is

$$
\begin{aligned}
& v_{r}=\Delta v \cdot \cos \left(\xi_{a}-\xi_{a}{ }^{\prime}\right)+v_{a} \cdot \sin \beta_{a} \\
& v_{\theta}=v_{a} \cdot \cos \beta_{a}-\Delta v \cdot \sin \left(\xi_{a}-\xi_{a}{ }^{\prime}\right)
\end{aligned}
$$

The speed after the orbit change is

$$
v_{a}{ }^{\prime}=\sqrt{v_{r}^{2}+v_{\theta}^{2}}
$$

The local velocity inclination angle after the orbit change is

$$
\beta_{a}^{\prime}=\frac{v_{r}}{v_{\theta}}
$$

Since the speed at that point after the orbit change is $v_{\alpha}^{\prime}$, the local velocity inclination angle $\beta_{a}^{\prime}$ and the orbit radius $r_{\alpha}$ are known, we could simulate the elliptical orbit after the orbit change.

- Parameter calculation after the orbit change

The debris travels on orbit $t$, the eccentricity $t$, major semi-axis and the perigee radius are:

$$
\begin{gathered}
e_{t}=\sqrt{\left[\left(r_{a} v_{a}^{\prime 2} / \mu\right)-1\right]^{2} \cos ^{2} \beta_{a}{ }^{\prime}+\sin ^{2} \beta_{a}{ }^{\prime}} \\
a_{t}=r_{a} /\left(2-\frac{r_{a} v_{a}^{\prime 2}}{\mu}\right) \\
r_{p t}=a_{t}\left(1-e_{t}\right)
\end{gathered}
$$

The orbit $t$ 's true anomaly and the eccentric anomaly at point $\alpha$ are:

$$
f_{a}=\arctan \frac{\left(r_{a} v_{a}^{\prime 2} / \mu\right) \sin \beta_{a}^{\prime} \cos \beta_{a}^{\prime}}{\left(r_{a} v_{a}^{\prime 2} / \mu\right) \cos ^{2} \beta_{a}^{\prime}-1}
$$




$$
E_{a}=2 \arctan \left(\sqrt{\frac{1+e_{t}}{1-e_{t}}} \tan \frac{f_{a}}{2}\right)
$$

We could discover the position of the orbit's major axis according to the true anomaly,

If $\tan f_{a}>0$, then the true anomaly is $\arctan \left(\tan f_{a}\right)$; if $\tan f_{a}<0$, then the true anomaly is $\pi+\arctan \left(\tan f_{a}\right)$.

Suppose that the pulse repetition frequency is $n$, then the pulse interval is $\frac{1}{n}$, the debris travels on the orbit $t$ and reaches point $\eta$ after the time of $\frac{1}{n}$. According to

$$
\frac{E_{\eta}-e_{t} \sin E_{\eta}-E_{a}+e_{s} \sin E_{a}}{\sqrt{\mu /\left(a_{t}^{3}\right)}}=\frac{1}{n}
$$

We could get $E_{\eta}$ ( $E_{\eta}$ is the eccentric anomaly of the orbit $t$ at point $\eta$ ), we could get $f_{\eta}$ according to the true anomaly and the eccentric anomaly's relationship ( $f_{\eta}$ is the true anomaly of orbit $t$ at point $\eta$ ). The orbit radius at point $\eta$ is

$$
r_{\eta}=a_{t}\left(1-e_{t} \cos E_{\eta}\right)
$$

We could get the zenith angle at point $\eta$ according to the geometrical relationship :

The calculation of pass times

$$
\begin{gathered}
\xi_{\eta}=\arctan \frac{r_{\eta} \sin \xi_{\eta}^{\prime}}{r_{\eta} \cos \xi_{\eta}^{\prime}-R} \\
\xi_{\eta}^{\prime}=\xi_{a}^{\prime}-\left|f_{a}-f_{\eta}\right|
\end{gathered}
$$

Step 1: Initialize the data. We know the elliptical orbit's eccentricity e and the perigee radius $r_{p}$, the orbit height $H$ at a certain point, the initial zenith angel $\xi$, the pulse repetition frequency $\mathrm{n}$ and the speed increment $\Delta v$ under the effect of the singular pulse laser.

Step 2: Calculate the major semi-axis a, the debris speed $\mathrm{v}$ and the local velocity inclination angle $\beta$.

Step 3: According to the debris material's ablation rate and the left ablation mass, we could calculate the speed increment $\Delta v$, and the elliptical orbit's parameters after the orbit change: orbit eccentricity $e^{\prime}$, major semi-axis $a^{\prime}$, orbit perigee radius $r_{p}^{\prime}$.

Step 4: Calculate the orbit height $H^{\prime}$, the speed $v^{\prime}$, the localvelocity inclination angle $\beta^{\prime}$, the true anomaly $f$, the eccentric anomaly $E$ at a certain point in the pulse interval $\left(\frac{1}{n}\right)$. The zenith angle at this time is $\xi^{\prime}$.

Step 5: Judge whether it requires a one pass, the judgment condition is: $\xi^{\prime} \leq 0$ and $r_{p}^{\prime}>6578 \mathrm{~km}$, if the conditions are met, go back to step 1 .

Step 6: Judge whether the removal is successful, the judgement condition is: $\xi^{\prime}>0$ and $r_{p}^{\prime} \leq 6578 \mathrm{~km}$, if the conditions are not met, go back to step 3.

Step 7: Collect the times of passes that is needed for removing the debris, and make a Table.

Table 2. For the debris at the height of 800 to $1500 \mathrm{~km}$

\begin{tabular}{cc}
\hline ablation times of the ps level lasers & Debris diameter interval \\
\hline 1 & {$[1,2.96]$} \\
2 & $(2.96,4.18]$ \\
3 & $(4.18,5.12]$ \\
4 & $(5.12,5.92]$ \\
5 & $(5.92,6.62]$ \\
6 & $(6.62,7.24]$ \\
7 & $(7.24,7.84]$ \\
8 & $(7.84,8.38]$ \\
9 & $(8.38,8.88]$ \\
10 & $(8.88,9.36]$ \\
11 & $(9.36,9.82]$ \\
12 & $(9.82,10]$ \\
\hline
\end{tabular}

According to the distribution, we discover that for every piece of debris, an average of 4.36 times of passes is needed to complete the removal. 
Table 3. For the debris at the height of 100 to $800 \mathrm{~km}$

\begin{tabular}{cc}
\hline Ablation times of the ps level lasers & Debris diameter interval \\
\hline 1 & {$[1,3.5]$} \\
2 & $(3.5,4.94]$ \\
3 & $(4.94,6.04]$ \\
4 & $(6.04,6.98]$ \\
5 & $(6.98,7.82]$ \\
6 & $(7.82,8.56]$ \\
7 & $(8.56,9.24]$ \\
8 & $(9.24,9.88]$ \\
9 & $(9.88,10]$ \\
\hline
\end{tabular}

According to the distribution, we discover that for every piece of debris, an average of 3.34 times of passes is needed to complete the removal.

According to Table 2 and Table 3, we can find when the size of a space debri is close to $10 \mathrm{~cm}$, a slight size change will cause the significant difference in the times of passes, so we do not consider using lasers to clean up the space debris with a diameter of more than $10 \mathrm{~cm}$.

\subsubsection{Cost Estimation of the Ground-Based Laser Method}

For the medium-sized space debris at the height of 100 to $1500 \mathrm{~km}$, we calculate the removal cost using the lasers in the following steps:

Step 1: Get the medium-sized debris' increment which is 3375 pieces per year according our MATLAB simulation.

Step 2: Convert the number of the debris into the volume of $23 \mathrm{~m} 3$.

Step 3: Calculate and get that there is $0.063 \mathrm{~m} 3$ of the waste is needed to be removed in order to maintain the increase of the waste.

Step 4: Calculate and get that $4 \mathrm{ps}$ level laser devices are needed according to the removing ability of the devices.

According to our calculation we discover that when using 4 ps level laser devices, the medium-sized space debris increased every year could just be removed.

Every laser device could work around 7 months and cost 1.5 million us dollars. With 4 devices works 40 times every day, it costs 210 thousand dollars per day to operate. Thus, it costs about 87.89 million dollars per year to operate the laser removal method.

\subsection{Cost Estimation of the High Pressure Water Jet}

According to the Figure about jet cutting pressure and cutting depth, we can conclude that water-jet can cut aluminum sheets with thickness of $1 \mathrm{~cm}$ when the cutting pressure is $100 \mathrm{mpa}$, i.e., it's enough to clear space debris. In the following calculations, we calculate the energy required to cut the space debris in the unit volume. So as to calculate the total amount of debris that the satellite can clean up every time, and then calculate the cost of cleaning space debris every year under the high pressure water-jet method.

\subsubsection{Parameters of Water Jet}

- Water jet exit velocity

According the Benulh equation and the conservation of energy, we get:

$$
g Z_{1}+\frac{P_{1}}{\rho}+\frac{1}{2} v_{1}^{2}=g Z_{2}+\frac{P_{2}}{\rho}+\frac{1}{2} v_{2}^{2}
$$

In the equation: $g$-gravity acceleration; $\rho$-density of water; $P_{l}$-pressure of the high-pressure water jet, $P_{2}$-pressure at the injector; $v_{1}$-average speed of the accumulator section; $v_{2}$ speed at the injector

Since $P_{1} \gg P_{2}, v_{2} \gg v_{1}$, we could suppose $P_{2}=0, v_{1}=0$ and get:

$$
\begin{aligned}
& P_{1}=\frac{1}{2} \quad v_{2}^{2} \\
& v_{2}=\sqrt{\frac{2 P_{1}}{\rho}}
\end{aligned}
$$

Also, $v_{\text {reality }}=\varphi v, \varphi$ is the energy converting coefficient of the injector, related to $\operatorname{Re}, \varphi=0.8 \sim 0.98$ 
As we could see, the jet's speed at the injector is only related to pressure, the jet's effect on objects is simply the conversion from pressure energy to kinetic energy.

- Water jet flow

$$
\begin{gathered}
Q_{\text {theory }}=A \cdot v=\frac{\pi}{4} d_{j}^{2} \cdot v=\frac{\pi}{4} \cdot d_{j}^{2} \sqrt{\frac{2 P}{\rho}} \\
Q_{\text {reality }}=C_{d} A \cdot v=C_{d} \cdot \frac{\pi}{4} \cdot d_{j}{ }^{2} \cdot v=C_{d} \cdot \frac{\pi}{4} \cdot d_{j}{ }^{2} \sqrt{\frac{2 P}{\rho}}
\end{gathered}
$$

In the equation, $C_{d}$ - jet flow coefficient at the injector (new injector $C_{d}=0.7$ ), which is related to the friction coefficient of the injector, $d_{j}$-injector diameter.

\section{- Water jet kinetic energy}

High pressure water jet cutting mainly depends on the kinetic energy $E$ of the water jet:

$$
E=\frac{1}{2} m v^{2}=\frac{1}{2} \rho Q v^{2}
$$

According to equation and the reality, we get:

$$
E=\sqrt{2} C_{d} \cdot A \cdot \varphi^{3} \cdot \rho^{-\frac{1}{2}} \cdot p^{\frac{3}{2}}
$$

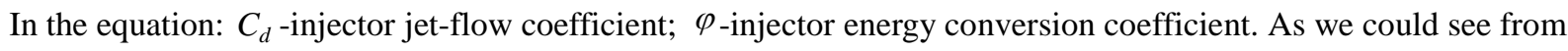
the equation (3-11), the jet's kinetic energy is proportion to the jet-flow and the $3 / 2$ power of the pressure. Thus, with the jet-flow fixed, increasing the pressure could result in higher kinetic energy.

\section{- Water jet power}

The injector is a component of the high pressure water jet's energy conversion system, the conversion efficiency could represent its performance. $\mu$ (conversion efficiency) is the power rate of the water jet produced by injector and the high-pressure water that enters the injector. Take one drop of water randomly in the injector. Because of the existence of the movement on $\mathrm{X}$-axis, the water drop takes no effect of the pressure $P$. Therefore the jet's energy is the jet's kinetic energy.

$$
\begin{gathered}
W_{\text {theory }}=\frac{1}{2} \rho Q_{\text {theory }} v_{0}^{2} \\
W_{\text {reality }}=\frac{1}{2} \rho Q_{\text {reality }} v_{1}^{2}
\end{gathered}
$$

Comparing the two equations above, we get that the conversion efficiency

$$
\mu=\frac{W_{\text {reality }}}{W_{\text {theory }}}=\frac{Q_{\text {reality }} v_{1}^{2}}{Q_{\text {theory }} v_{0}^{2}}=C_{d}^{3}
$$

which reflect the power loss in some extend.

\subsubsection{Calculation of the Cost of Removing Debris by the Method of Water Jet}

Step 1: Calculate and get that the available water capacity is $1.3 t$ according to the spaceships' capacity.

Step 2: According to the Figure about water-jet cutting pressure and cutting depth, get that when the jet cutting pressure is $100 \mathrm{MPa}$ and water jet speed is $450 \mathrm{~m} / \mathrm{s}$, the cutting of the space debris is available.

Step 3: According to the kinetic energy theory, calculate and get that the total energy needed for injecting all the water is $W_{\text {reality }}=219375 k j$, the energy of the emitted water is $W_{\text {theory }}=131625 k j$, calculate and get the the conversion efficiency $\mu$ is $60 \%$.

Step 4: According to the mass of the water needed for every cut, calculate and get that the total cutting time possible using all the water is 92198581 .

Step 5: Knowing that it needs 1 million times to completely remove the debris per unit volume, calculate and get that $92.1 \mathrm{~m} 3$ of the waste could be removed per flight of the spaceship.

Step 6: According to the distribution Table of the increment and the size of the large-sized debris, calculate and get that the approximate volume of the increasing large-sized debris is $20.69 \mathrm{~m} 3$.

Comparing the step 5 and 6 , get that every flight per year of the water jet spaceship could not only remove the increasing debris, but also remove the existing 750 pieces. The cost of every year is around 300 million dollars. 


\subsection{Cost Estimation of the Space Net-Capture Method}

The casting parameter of the space net-capture directly affects the success rate of the capturing, and therefore affects the space net-capture's economy benefits. In order to explore deeper into how the parameter specifically affect the net's casting success rate, we build a shifting model of the net's casting.

\section{- Node Caculation}

Build the kinetic balance equation at the initialization of time increment step:

$$
M \ddot{u}=P-I
$$

In the equation: $M$ stands for the mass matrix of the node; $\ddot{u}$ stands for the acceleration of the node; $P$ stands for the external force cast on the node; $I$ stands for the unit internal stress.

$$
\left.\ddot{u}\right|_{(t)}=\left.(M)^{-1} \cdot(P-I)\right|_{(t)}
$$

Integrate the acceleration using the method of central differences at time, when the increment step is taken very small, the acceleration $\left.\ddot{u}\right|_{(t)}$ in the increment step could be seen as a constant, use this speed change value and the previous increment step's central point's speed $\dot{u}_{\left(t-1-\frac{\Delta t}{2}\right)}$ to get the central point's speed:

$$
\left.\dot{u}\right|_{\left(t+\frac{\Delta t}{2}\right)}=\left.\dot{u}\right|_{\left(t-\frac{\Delta t}{2}\right)}+\frac{\left(\left.\Delta t\right|_{(t+\Delta t)}+\left.\Delta t\right|_{(t)}\right)}{2} \ddot{u} \mid \omega
$$

By adding the displacement $\left.u\right|_{\omega}$ to the integration of speed to time, get the displacement when the increment step ends:

$$
\left.u\right|_{(t+\Delta t)}=\left.u\right|_{\omega}+\left.\left.\Delta t\right|_{(t+\Delta t)} \dot{u}\right|_{\left(t+\frac{\Delta t}{2}\right)}
$$

\section{- Parameter Calculation}

In the process of the net casting, the net spreads through the towing piece, thus the mass of the piece $m_{s}$, the speed of the casting $v_{0}$ and the casting angle $\theta$ are the keys of the success rate of the net casting. The casting angle defines the towing piece's casting direction and the casting direction angle.

According to the kinetic equation, we use MATLAB to calculate the optimum solution of the mass $m_{s}$, the casting speed $v_{0}$ and the casting angle $\theta$.

As known in the reference, invalid capturing distance and the distance of spreading to the predicted position mainly depend on the towing piece's mass $m_{s}$. The time of spreading to the predicted position and the distance of spreading to the maximum area mainly depend on the casting speed $v_{0}$. The casting angle $\theta$ is the key factor in affecting the net's spreading area at the predicted position. The towing piece's mass and the casting speed's increasing is beneficial for the net's spreading, making the net gain extra spreading area and speed, reach the predicted position faster and higher the success rate.

Invalid distance decreases first and increases later and has the minimum value. Because of the net's spreading, the casting parameters' changing has waves. Therefore in the ranges of the parameters given by the industry, we search for the valid spreading's optimal parameter combine. Set the capture distance at $\mathrm{C}=30 \mathrm{~m}$.

Keep the casting speed $v_{0}$, the casting angle $\theta$ unchanged, change the towing piece's mass $m_{s}$, explore their influence on the net's casting results, as shown in the Figure 5 and 6.

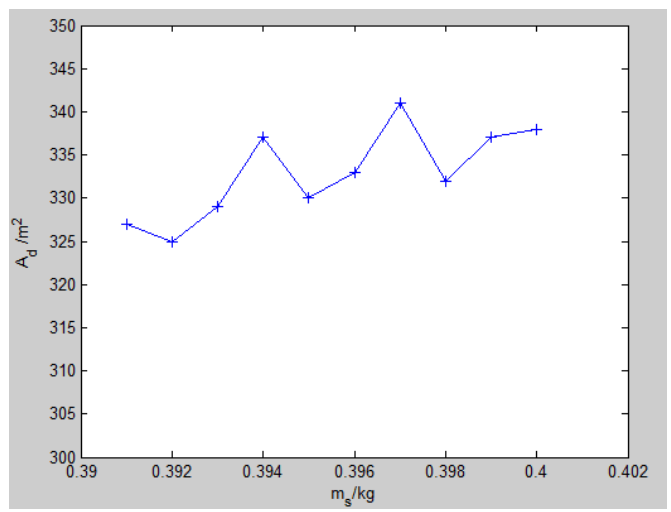

Figure 5. The towing piece's mass' influence on the spreading area 


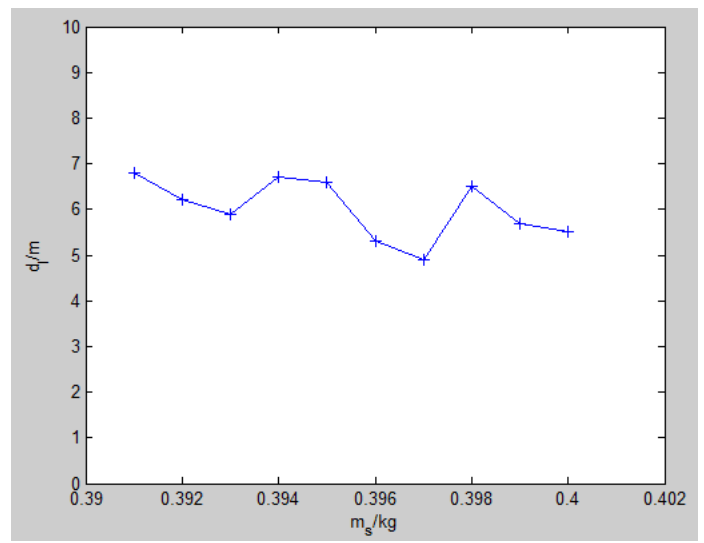

Figure 6. The towing piece's mass' influence on the invalid capture distance

As we could see in the Figure 5 and 6, when the mass $m_{s}=0.397 \mathrm{~kg}$, the spreading area is at maximum, and the invalid capture distance is at the minimum.

Keep the mass $m_{s}$, the casting angle $\theta$ unchanged, change the speed $v_{0}$ and explore its influence on the casting results, as shown in the Figure 7 and 8.

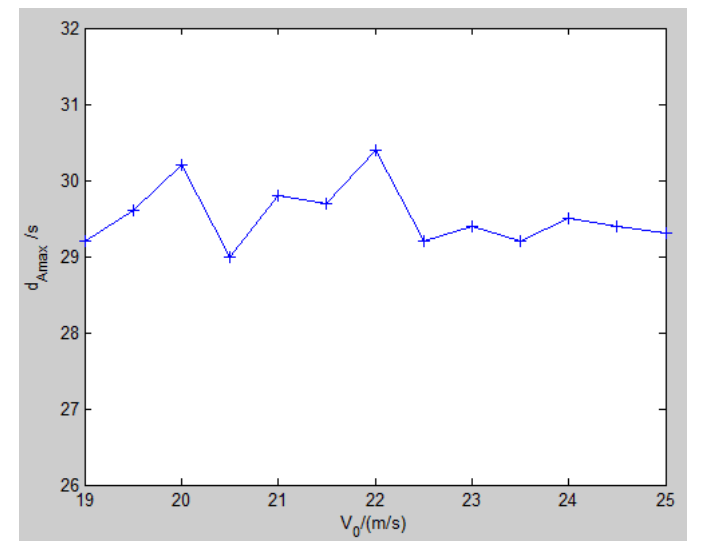

Figure 7. The casting speed's influence on the distance at maximum area

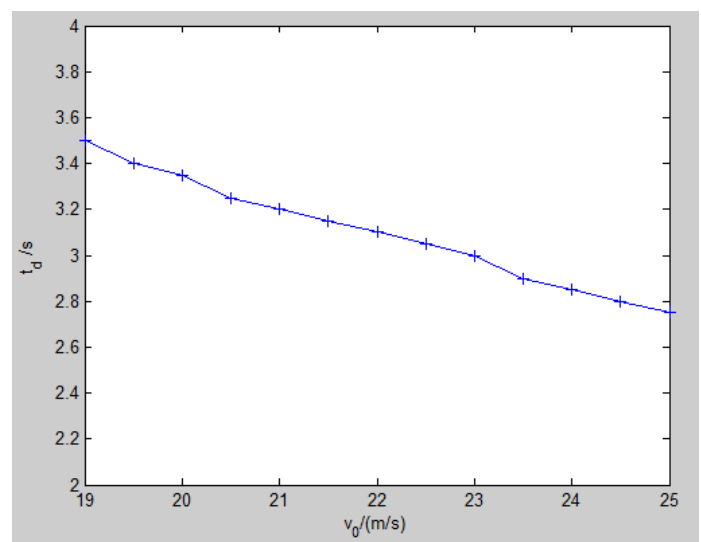

Figure 8. The casting speed's influence on the time at predicted position

As we could see in the Figure 7 and 8 , when the casting speed $v_{0}=22 \mathrm{~m} / \mathrm{s}$, the net's distance at maximum area reaches furthest, and the time cost is relatively smaller.

Keep the mass $m_{s}$, casting speed $v_{0}$ unchanged, change the casting angle $\theta$ and explore its influence on the casting result, as shown in the Figure 9. 


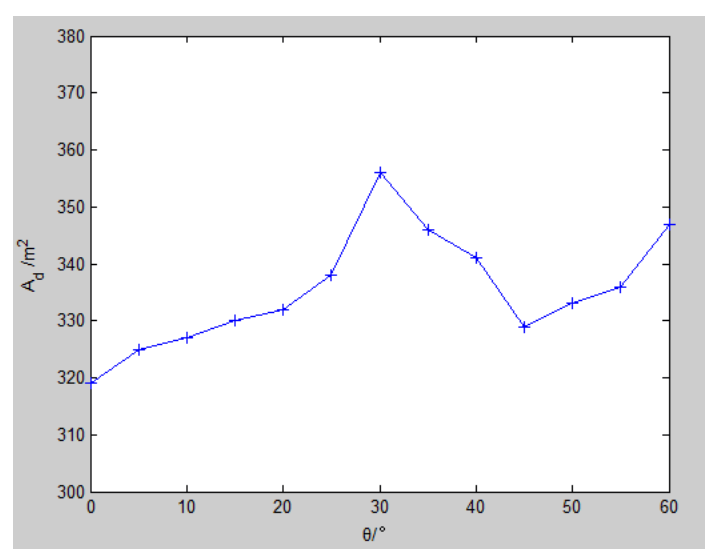

Figure 9. The casting angle's influence on the spreading area

The towing piece's casting angle $\theta$ also influence the net's spreading, but is not a key factor, it mainly decides the spreading area at the capture distance and takes little influence from other parameters in the simulation process. The Figure 9 shows that when $\theta=30^{\circ}$ the net reaches the maximum spreading area, thus the best casting angle is $30^{\circ}$.

By analyze the mass $m_{s}$, the casting speed $v_{0}$ and the casting angle $\theta$ 's influence on the net casting, we get that the optimal results is mass $m_{s}=0.397 \mathrm{~kg}$, casting speed $v_{0}=22 \mathrm{~m} / \mathrm{s}$, casting angle $\theta=30^{\circ}$.

We think the capture success rate reaches $100 \%$ only under the circumstances of the optimal results.

- Cost estimation using the net-capture method

Knowing that the cost for launching every satellite is 4.9 million dollars and building every removing satellite is 140 thousand dollars. Calculate the satellite's launching and building cost, every removal cost about 5.04 million dollars.

Since the net-capture method is a kind of suicide removal and every satellite could only bring one space debris, when using the net-capture method, no matter what size the debris is, the cost stays at 5.04 million dollars. Therefore the net-capture method doesn't stand out when removing the medium-sized and small-sized debris. It would cost too much only use the net-capture method singly.

\section{Conclusion}

The threat of the space debris' rapid increase does to the space industry is getting bigger. The significance of removing the debris is also getting bigger.

First we got the best plans at removing the space debris. Since we didn't have the data for the space debris, We could know that the debris pieces are rapidly increasing 5\% per year according to our simulation model. We assumed that the space debris were round aluminum plates thick $1 \mathrm{~cm}$, then we analyzed the three common methods in removing the space debris (ground-based laser, water jet cutting and net-capture), and got the pros and cons of each method:

Ground-based laser: Appropriate for removing medium-sized debris pieces. The cost of removing pieces with diameter shorter than $10 \mathrm{~cm}$ and longer than $1 \mathrm{~cm}$ would be less, and would rise rapidly when removing pieces with diameters longer than $10 \mathrm{~cm}$. However, it has lower risks since it's operated on earth's surface.

Water jet cutting: Appropriate for removing large-sized debris pieces with diameters between $10 \mathrm{~cm}-1400 \mathrm{~cm}$. There would be waste of resource when removing the medium-sized pieces shorter than $10 \mathrm{~cm}$. The water jet spaceship could remove around 1,001 pieces of large-sized debris per flight, which is quite a high usage rate. Because the operating environment is in space, it has a higher risk.

Net-capture: Appropriate for super-large-sized debris pieces. Since it could only bring one piece of the debris, no matter what size it is, it's a waste using it to remove the small-sized pieces. But it has a higher usage rate when removing the super-large-sized debris pieces.

\section{References}

Apollonov, V. V. (2013). High power lasers for space debris elimination. Chinese Optics, 2013. https://doi.org/10.3788/co.20130602.0187 
Bin, T. (2003). Research on the theory and technology of high pressure water jet processing. Dalian University of Technology, 2003.

Chunlai, L., Ziyuan, O., \& Heng, D. (2002). Space debris and space environment. Quaternary Sciences, 6, 540-551.

Gensheng, L., Hualin, L., Zhongwei, H., Zhonghou, S. (2009). Rock damage and fracture mechanism under the action of super high pressure water jet. Journal of Mechanical Engineering, 10, 284-293.

Guoliang, T. (2008). Present situation of space debris and future. Modern Physics, 2, 36-41.

Hao, C., Xing, J., Yanji, H., \& Xiuqian, L. (2012). Modeling and Simulation of debris process in the ground laser cleaning process. Journal of Aeronautical Science, 6, 994-1001.

Hongjian, N., Ruihe, W., \& Hongkui, G. (2004). High pressure water jet rock breaking numerical simulation analysis. Chinese Journal of Rock Mechanics and Engineering, 4, 550-554.

Jingyang, L., Yang, Y., \& Yinhexi, B. (2012). Net optimization system parameter space projection. Journal of Astronautics, 6, 823-829.

Juliang, J., Jianqiang, L., Yuliang, Z. et al. (2014). The preliminary theoretical framework of disaster assessment of drought risk. Journal of Catastrophology, (3), 1-10.

Ruihe, W., \& Hongjian, N. (2002). Tthe rock breaking mechanism: Study on high pressure water jet. Journal of the University of Petroleum (NATURAL SCIENCE EDITION), 4, 118-122.

Schmitz, M., Fasoulas, S., \& Utzmann, J. (2015). Performance Model for Space-Based Laser Debris Sweepers. Acta Astronautica, 115, 376-383. https://doi.org/10.1016/j.actaastro.2015.05.032

Wenhui, Z. (2008). Numerical simulation of internal and external flow field of high pressure water jet nozzle. Lanzhou University of Technology, 2008.

Yang, Y., Yinhexi, B., \& Junfeng, L. (2010). Space net expansion of projectile dynamics modeling and Simulation of space.

Yilin, Z. (2000). Modeling and analysis of space debris environment in low earth orbit. Shanghai Aerospace, 3, $18-22$.

\section{Copyrights}

Copyright for this article is retained by the author(s), with first publication rights granted to the journal.

This is an open-access article distributed under the terms and conditions of the Creative Commons Attribution license (http://creativecommons.org/licenses/by/4.0/). 